\title{
CEREBRAL ISCHEMIC EVENTS WITH SICKLE CELL ANEMIA
}

Researchers at Cincinnati Children's Hospital and several additional centers in the US and UK studied the incidence of acute silent cerebral ischemic events (ASCIEs) in MRIs of children with asymptomatic sickle cell anemia (SCA). ASCIEs were detected in $1.3 \%$ of MRIs ( 10 of 771) in 652 children (mean age 10 years), with an incidence of 47.3 events per 100 patient-years. At follow-up MRIs in 2 of 10 children with ASCIEs, only 1 had silent cerebral infarction correlating with the location of a previously detected ASCIE. Children with SCA are at risk of frequent ongoing chronic, intermittent, cerebral ischemic events, sometimes reversible. (Quinn CT, McKinstry RC, Dowling MM, et al. Acute silent cerebral ischemic events in children with sickle cell anemia. JAMA Neurol 2013 Jan;70(1):58-65). (Response: Michael R DeBaun MD, Vanderbilt Meharry Center for Sickle Cell Disease Excellence, 220 Children's Way, Rm 11206 DOT, Nashville, TN 37232. E-mail: m.debaun@vanderbilt.edu).

COMMENT. The authors conclude that children with SCA experience clinically silent cerebral ischemia far more frequently than previously recognized. The brain in SCA is at constant threat of ischemic injury. The Nashville experience with neurologic injury in SCA finds that the specific morbidity includes a decrement in general intellectual abilities, poor academic achievement, progression in overt stroke, and new SC infarct despite regular blood transfusion therapy. (De Baun MR et al. Blood 2012 May 17;119(20):4587-96).

\section{ISCHEMIC STROKE AND TIA IN YOUNG ADULTS}

Researchers at the Massachusetts General Hospital, Boston, MA studied retrospectively the risk factors and yield of diagnostic tests, neuroimaging findings, and treatment of ischemic strokes in 215 consecutive young adult patients (aged 18 to 45 years) seen between 2005 and 2010. Hypertension was recorded in 20\%, diabetes mellitus in $11 \%$, dyslipidemia in $38 \%$, and smoking in $34 \%$. Cerebral angiography abnormalities were relevant in $67 \%$, cardiac ultrasonography was abnormal in $50 \%$, Holter monitoring in $1 \%$, and hypercoagulable panel in $16 \%$. Multiple infarcts observed in $31 \%$ were more prevalent in $<35$ year-olds. Arterial lesions occurred in the middle cerebral artery in $23 \%$, internal carotid in $13 \%$, and vertebrobasilar arteries $13 \%$. Cardioembolic stroke occurred in $47 \%$, including $17 \%$ with patent foramen ovale. Outcome was good in $81 \%$ at discharge. Of 29 patients receiving short-term thrombolysis, 55\% had a good outcome at hospital discharge, and none had symptomatic brain hemorrhage. (Ji R, Schwamm LH, Pervez MA, Singhai AB. Ischemic stroke and transient ischemic attack in young adults. Risk factors, diagnostic yield, neuroimaging, and thrombolysis. JAMA Neurol 2013;70(1):51-57). (Response: Aneesh B Singhal MD, ACC-729C, Department of Neurology, Massachusetts General Hospital, Boston, MA 02114. E-mail: asinghal@partners.org).

COMMENT. Advances in thrombolysis are discussed in the 2012 Stroke Roundup (Kaste M. Lancet Neurol 2013 Jan;12(1):2-4). Based on the Safe Implementation of 
Treatments in Stroke registries, the Virtual International Stroke Trials Archive, and the US FDA, age alone is not a barrier to thrombolysis with alteplase in adults, but patients over 80 years of age are excluded by European Regulatory Authorities. The Third International Stroke Trial revealed a significantly greater treatment effect of alteplase in patients over 80 years of age than in those aged 80 or younger. Alteplase treatment within 6 hours of stroke onset improved functional outcome. A risk of symptomatic intracerebral hemorrhage with alteplase is a deterrent to more frequent use, but further studies are indicated, especially in younger patients.

A PubMed search of the literature uncovers several single case reports of the successful use of thrombolysis in the treatment of childhood stroke. (Condie J, Shaibani A, Wainwright MS. Successful treatment of recurrent basilar artery occlusion with intraarterial thrombolysis and vertebral artery coiling in a child. Neurocrit Care 2012 Feb;16(1):158-62). Coordination of neurology, critical care, and interventional radiology is recommended in management of ischemic stroke in children.

Currently, there are two treatment guidelines for therapy of childhood AIS (Royal College of Physicians in the UK, and American Heart Association and American College of Chest Physicians). Antithrombotic strategies are advocated, but the choice of agent varies with the guideline preference and risk factors. Thrombolytic treatment is not advocated. (Eleftheriou D, Ganesan V. Treatment strategies for childhood stroke. Expert Opin Pharmacother 2008 Dec;9(17):2955-67). In a multicenter, observational, cohort study, alteplase was used infrequently and the outcome was often poor; intracranial hemorrhage occurred in 4 of 15 patients. (Amlie-Lefond C, et al. International Pediatric Stroke Study. Use of alteplase in childhood arterial ischaemic stroke. Lancet Neurol 2009 Jun;8(6):530-6). Clinical trials are needed to determine optimal dose of alteplase in childhood stroke.

\section{DEVELOPMENTAL DISORDERS}

\section{HEMIMEGALENCEPHALY: A FOETAL TAUOPATHY}

Researchers at University of Calgary and Alberta Children's Hospital, Canada; and University of Pennsylvania, Philadelphia studied brain resections from 3 male infants with hemimegalencephaly (HME) and refractory epilepsy. One infant died at 2.5 months, and one has Proteus syndrome. The hippocampus and neocortex of HME showed cytoarchitectural abnormalities on electronmicroscopy and intense tau immuno-reactivity. The post-mortem non-HME hemisphere had sparse dysmorphic tau-reactive cortical neurons but none in subcortical regions. Numerous enlarged and dysmorphic cells exhibited immunoreactivity indicative of mTOR activation. Tau expression and mTOR activation were absent in control brains. Electron microscopy in each case showed lipid in neurons and lipid storage by light microscopy. The investigators propose that the pathogenesis of HME may involve an early defect in microtubules, probably related to the AKT3 gene. Lipidosis of neurons and glia suggests a metabolic impairment of undetermined type, related to tauopathy in HME. Perinatal treatment with everolimus (rapamycin), an inhibitor of mTOR pathway, might reduce the morbidity of HME, including epilepsy. (Sarnat H, Sarnat LF, Crino P, Hader W, Bello-Hespinosa L. Hemimegalencephaly: foetal tauopathy with mTOR hyperactivation and neuronal 